Article

\title{
Direct Amplification of High Energy Pulsed Laser in Fiber-Single Crystal Fiber with High Average Power
}

\author{
Feng $\mathrm{Li}^{1}{ }^{1,2, *} \mathbb{C}$, Zhi Yang ${ }^{1, *}$, Zhiguo $\mathrm{Lv}^{1}$, Yang Yang ${ }^{1}$, Yishan Wang ${ }^{1,2}$, Xiaojun Yang ${ }^{1}$, \\ Wei Zhao ${ }^{1,2}$, Qianglong $\mathrm{Li}^{1}$ and Yufeng Wei ${ }^{1}$ \\ 1 State Key Laboratory of Transient Optics and Photonics, Xi'an Institute of Optics and Precision Mechanics, \\ Chinese Academy of Sciences, Xi'an 710119, China; lvzhiguo@opt.ac.cn (Z.L.); laser_yangyang@opt.cn (Y.Y.); \\ yshwang@opt.ac.cn (Y.W.); yxj@opt.ac.cn (X.Y.); 1f5322@163.com (W.Z.); laser_liqianglong@opt.cn (Q.L.); \\ laser_weiyufeng@opt.cn (Y.W.) \\ 2 Institute Collaborative Innovation Center of Extreme Optics, Taiyuan 030006, China \\ * Correspondence: laser_lifeng@opt.cn (F.L.); yangzhi@opt.ac.cn (Z.Y.)
}

Received: 22 March 2019; Accepted: 19 April 2019; Published: 21 April 2019

\begin{abstract}
A laser master oscillator power amplifier (MOPA) system consisting of a fiber amplifier and a two-stage Yb:YAG single crystal fiber (SCF) is experimentally studied. The nonlinear stimulated Raman scattering (SRS) is avoided by limiting the output power of the fiber preamplifier to $600 \mathrm{~mW}$. Due to the benefit from the low nonlinearity and high amplification gain of the SCF, a laser pulse duration of $16.95 \mathrm{ps}$ and a high average power of $41.7 \mathrm{~W}$ at a repetition rate of $250 \mathrm{kHz}$ are obtained by using a two-stage polarization controlled double-pass amplification of Yb:YAG SCF, corresponding to an output energy of $166.8 \mu \mathrm{J}$ and a peak power of $9.84 \mathrm{MW}$, respectively. The polarization controlled SCF amplification scheme achieved a gain as high as more than 69 times. During the amplification, the spectra gain narrowing effect and the polarization controlled four-pass amplification setup are also studied. The laser spectrum is narrowed from over $10 \mathrm{~nm}$ to less than $3 \mathrm{~nm}$, and the pulse width is also compressed to hundreds of femtosecond by dechirping the laser pulse. This compact-sized, cost-effective laser source can be used in laser micromachining, or as the seeder source for generating much higher power and energy laser for scientific research. For some applications which need femtosecond laser, this laser source can also be compressed to femtosecond regime.
\end{abstract}

Keywords: single crystal fiber; ytterbium doped fiber amplifier; high-energy ultrashort laser amplification

\section{Introduction}

High-performance picosecond lasers with high-power, high-energy, and high-repetition-rate are in great demand in many industrial micromachining [1,2] and scientific applications [3], such as the processing of ceramics, diamonds, certain transparent materials, and optical parametric chirped pulse amplification (OPCPA) systems. Different kinds of laser amplifiers are used to obtain the high-power, high-energy picosecond laser. With the appearance of large mode area photonic crystal fiber $[4,5]$, the influence of the nonlinear effect in the fiber laser amplifier is reduced. Fiber amplifiers have potential in high-power picosecond laser amplification, and by using the fiber master oscillator power amplifier (MOPA) system, some high-power picosecond MOPA systems are demonstrated. Ho-Yin Chan et al. achieved laser power of $97 \mathrm{~W}$ and energy of $17.7 \mu \mathrm{J}$ with pulse duration of 124 ps by using a four-stage fiber MOPA [6]. S. Pierrot reported $83 \mathrm{~W}, 14 \mu \mathrm{J}, 5.9 \mathrm{MHz}, 30 \mathrm{ps}$ MOPA fiber laser by using a rod-type large mode area photonic crystal fiber [7]. Tong Liu et al. used a multi-stage fiber MOPA to obtain an average power of $117 \mathrm{~W}$, pulse width of $200 \mathrm{ps}$ at a repetition rate of $25 \mathrm{MHz}$ [8]. The fiber MOPA system can obtain high-power output, however, due to the high nonlinearity during the amplification the peak power is limited, typically no more than $1 \mathrm{MW}$. The thin disk Yb:YAG laser and Yb-Innoslab laser 
achieved some impressive results, such as 1.6 ps pulses with $30 \mathrm{~mJ}$ at $300 \mathrm{~W}$ of output power by using a thin disk laser [9], and $54 \mathrm{~mJ}, 1.5 \mathrm{ps}$ at $0.5 \mathrm{~kW}$ of output power by using a Innoslab laser [10]. However, its inherent complexity and thermal management pose a challenge for many applications. Therefore, some relatively simple systems are studied, such as using the double-passing end-pumped Nd:YVO4 rod amplifier [11], which delivers $90 \mathrm{ps}$ and $10.2 \mathrm{~W}$ laser at the repetition of $70 \mathrm{kHz}$ with a peak power of 1.6 MW. By using the fiber and bulk hybrid MOPA system, a $70 \mathrm{~W}$ average power laser at $1064 \mathrm{~nm}$ with $1000 \mathrm{kHz}$ repetition rate and 83 ps pulse duration is demonstrated [12].

The SCF is a new architecture, between the bulk crystals and the optical fibers, allowing higher average powers than with conventional crystals, and higher energy than with optical fibers in a pulsed regime. During the SCF amplification, the pump laser from the fiber-coupled laser diode is confined by the guiding capacity of the Yb:YAG SCF, whereas the seeder laser beam is in free propagation. Due to the benefit from the better overlap between the pump beam and signal beam, a higher amplification gain can be obtained. Therefore, this technology has prospects in picosecond or femtosecond laser systems with compact size and simplicity, and by employing this kind of amplifier the systems are more robust and less expensive. To generate femtosecond laser, the single crystal fiber is employed in chirped pulse amplification systems [13], and it is also demonstrated in a direct picosecond laser amplification, which has typical output parameters of $200 \mathrm{k} \mathrm{Hz}, 180 \mu \mathrm{J}$. The seeder is a high-energy commercial laser source with energy of $40 \mu \mathrm{J}$ and pulse width of $27 \mathrm{ps}$ [14].

As in our previous work, we focus on the high-energy femtosecond laser. By using the SCF in the chirped amplification system, we obtained average power of $15.57 \mathrm{~W}$, pulse duration of $715 \mathrm{fs}$ and pulse energy of $155.7 \mu \mathrm{J}$. During the amplification, the pulse is stretched to more than 600 picosecond and uses the transmission grating pair to compress the pulse [15]. Due to the short pulse duration, we can achieve so called "cold processing" in the applications of high-precision laser micromachining, such as materials cutting, etching, and hole drilling. However, we have to balance the heat effect and the processing efficiency. Specifically, the shorter pulse width results in less thermal effect in the processing, however, the laser source will be more expensive with the dispersion components. With the same cost, we can obtain a higher high-power picosecond laser with high processing efficiency. In many applications, the thermal effect in the processing is acceptable with a laser pulse width of tens of picosecond. In this work, we report a high-energy and high-power picosecond laser with fiber-single crystal fiber hybrid MOPA system by employing two-stage SCF amplifiers, which delivers a maximum output $41.7 \mathrm{~W}$ at $250 \mathrm{kHz}$, with a pulse width of $16.95 \mathrm{ps}$, corresponding to a peak power of $9.84 \mathrm{MW}$, and the spectrum width is narrowed to $\sim 3 \mathrm{~nm}$. The correlation between the spectra revolution and the pulse width is also explored. The polarization controlled high-gain four-pass amplification setup is also experimentally studied, which can obtain higher gain as compared with the single- and double-pass amplification setup.

\section{Experimental Setup}

The experimental setup, schematically shown in Figure 1, consisted of an oscillator, a $100 \mathrm{~m}$ polarization maintaining (PM) fiber stretcher, a one-stage polarization maintaining (PM) single-mode fiber and two-stage PM cladding fiber amplifier, a pulse picker, and a two-stage Yb:YAG SCF amplifier.

The seed source was a mode-locked PM fiber laser operating at $40.7 \mathrm{MHz}$ repetition rate, delivering $10 \mathrm{ps}$ pulses centered at $1030 \mathrm{~nm}$ with spectral bandwidth of $10 \mathrm{~nm}$ and average power of $20 \mathrm{~mW}$. This was a positive chirped pulse output and could be compressed to less than $300 \mathrm{fs}$. The $100 \mathrm{~m}$ PM fiber stretcher was utilized to stretch the pulse duration to about $40 \mathrm{ps}$, to lower the nonlinearity accumulated in the fiber amplifier stage. The first stage amplifier was a single mode amplifier, and an output power of $80 \mathrm{~mW}$ was obtained when the pump power was $250 \mathrm{~mW}$. The second amplifier was a double cladding PM Yb-doped fiber (YDF) amplifier with a core diameter of $10 \mu \mathrm{m}$ and a cladding diameter of $125 \mu \mathrm{m}$ with fiber length of $1.2 \mathrm{~m}$. At a pump power of $4.5 \mathrm{~W}$, the output power of $1.5 \mathrm{~W}$ was obtained. Then, a fiber-based acousto-optic modulator was used to decrease the repetition rate of the oscillator before amplification in the third stage double cladding YDF amplifier with a core 
diameter of $25 \mu \mathrm{m}$ and cladding diameter of $250 \mu \mathrm{m}$ with fiber length of $1.5 \mathrm{~m}$. The repetition rate was decreased to $250 \mathrm{kHz}$ in the second stage fiber amplifier. After the AOM, the average power was approximately $2 \mathrm{~mW}$. Limited by the severe nonlinearity, especially the onset of the nonlinear stimulated Raman scattering, the amplified power was limited to about $600 \mathrm{~mW}$.

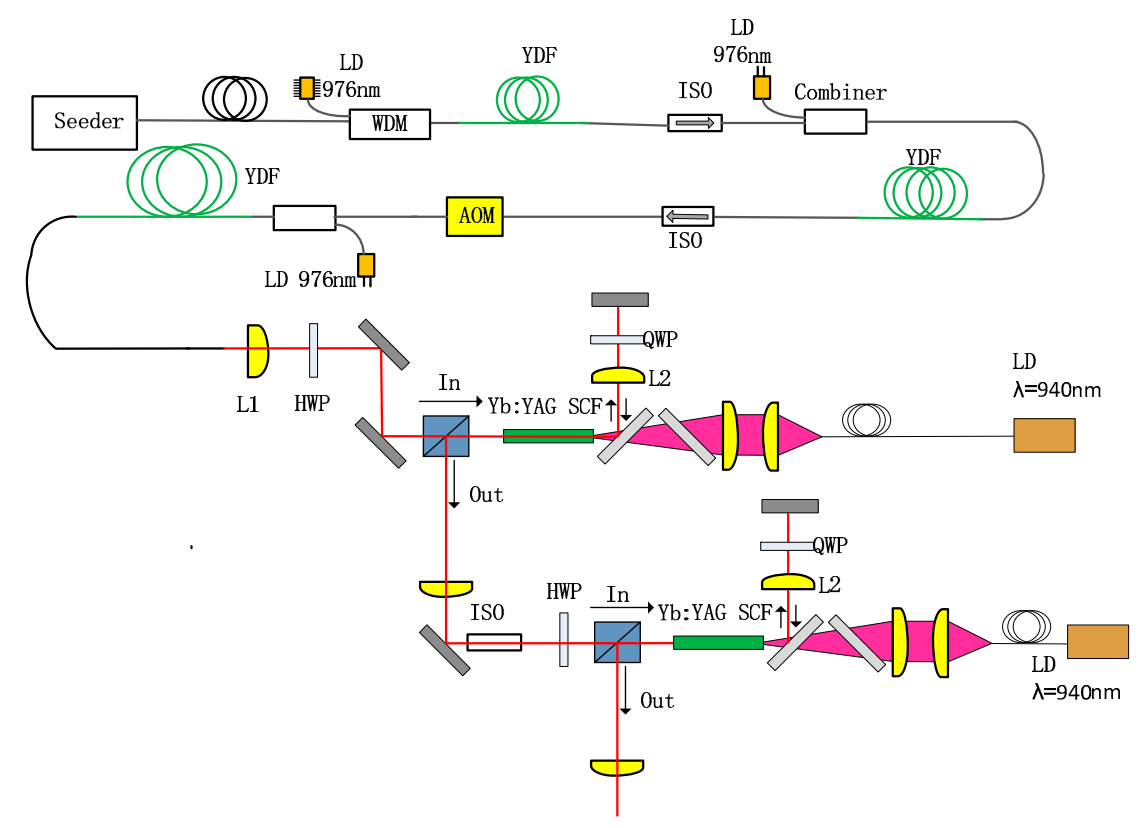

Figure 1. Schematic diagram of the high energy picosecond master oscillator power amplifier (MOPA) system. WDM: wavelength-division multiplexer; LD: laser diode; YDF: Yb-doped fiber; HWP: half wave plate; QWP: quarter wave plate; ISO: isolator

The main amplifier was a two-stage polarization controlled double-pass amplifier based on the Yb:YAG SCF gain module using the parameters of $30 \mathrm{~mm}$ length, diameter of $1 \mathrm{~mm}$, and doping rate of 1 at. \%. The facets were antireflection coated for both the signal and the pump wavelength to prevent parasitic lasing and excess losses at the interfaces. The SCF module was water cooled at a temperature of $20^{\circ} \mathrm{C}$ by a water cooler with a cooling capacity of $1300 \mathrm{~W}$. During the amplification, the seed beam from the preamplifier with core diameter of $25 \mu \mathrm{m}$ was collimated to $\sim 0.4 \mathrm{~mm}$ and the diameter was further propagated through Yb:YAG SCF. The SCF was end pumped by a fiber coupled laser diode with an output power of $140 \mathrm{~W}$ at $940 \mathrm{~nm}$, a core diameter of $106 \mu \mathrm{m}$, and an NA of 0.22 . The pump was imaged inside the SCF with a magnification factor of 3.75 using two plano convex lenses. The focused pumped laser beam diameter was about $400 \mu \mathrm{m}$. A pair of dichroic mirrors with parameters of reflectivity of $99.5 \% @ 1030 \mathrm{~nm}$ and transmissivity of $97 \% @ 1020-1080$ nm was used to separate the incoming pump beam and the signal beam. After the first pass amplification, the laser beam was focused by a lens (L2), and its laser waist was located on a highly reflective mirror coating at 0 degree consisting a $\mathrm{f}-\mathrm{f}$ configuration. A quarter wave plate was inserted before the reflective mirror. After the laser beam passed the quarter wave plate twice, its polarization direction was rotated by $90^{\circ}$ and could be extracted by the polarization beam splitter (PBS). After the first stage SCF amplifier, the power was about $12 \mathrm{~W}$. Then the amplified laser was injected into the second stage SCF amplifier with a plano-convex lens with a focal length of $250 \mathrm{~mm}$ and the double-pass structure was nearly the same as the first stage amplifier.

\section{Experimental Results and Discussions}

In the fiber preamplifier, the nonlinearity, especially the stimulated Raman scattering, is avoided. In the second stage double cladding fiber amplifier, the pulse repetition rate is only $250 \mathrm{kHz}$. In order to generate high peak power laser confined in the $25 \mu \mathrm{m}$ fiber core, the nonlinear self-phase modulation 
and the stimulated Raman scattering is found. By controlling the amplification gain, different output power and the corresponding spectrum is detected, as shown in Figure 2. The spectra of amplified power of $220 \mathrm{~mW}, 525 \mathrm{~mW}, 600 \mathrm{~mW}$, and $700 \mathrm{~mW}$ are measured using the optical spectrum analyzer (AQ 6370C, YOKOGAWA, Tokyo, Japan), respectively, from the spectra. In order to avoid the onset of the SRS, the amplified power is limited to less than $600 \mathrm{~mW}$. During the fiber amplification, the spectrum is carefully detected, because the ASE peak is the same wavelength centered in $1030 \mathrm{~nm}$ in the fiber amplifier, and therefore it is hard to assess the amount of ASE in the preamplifier. However, the ASE have different laser divergence as compared with the laser beam, so we detected the ASE using an infrared card since after the laser output from the fiber, the ASE component could not be detected.
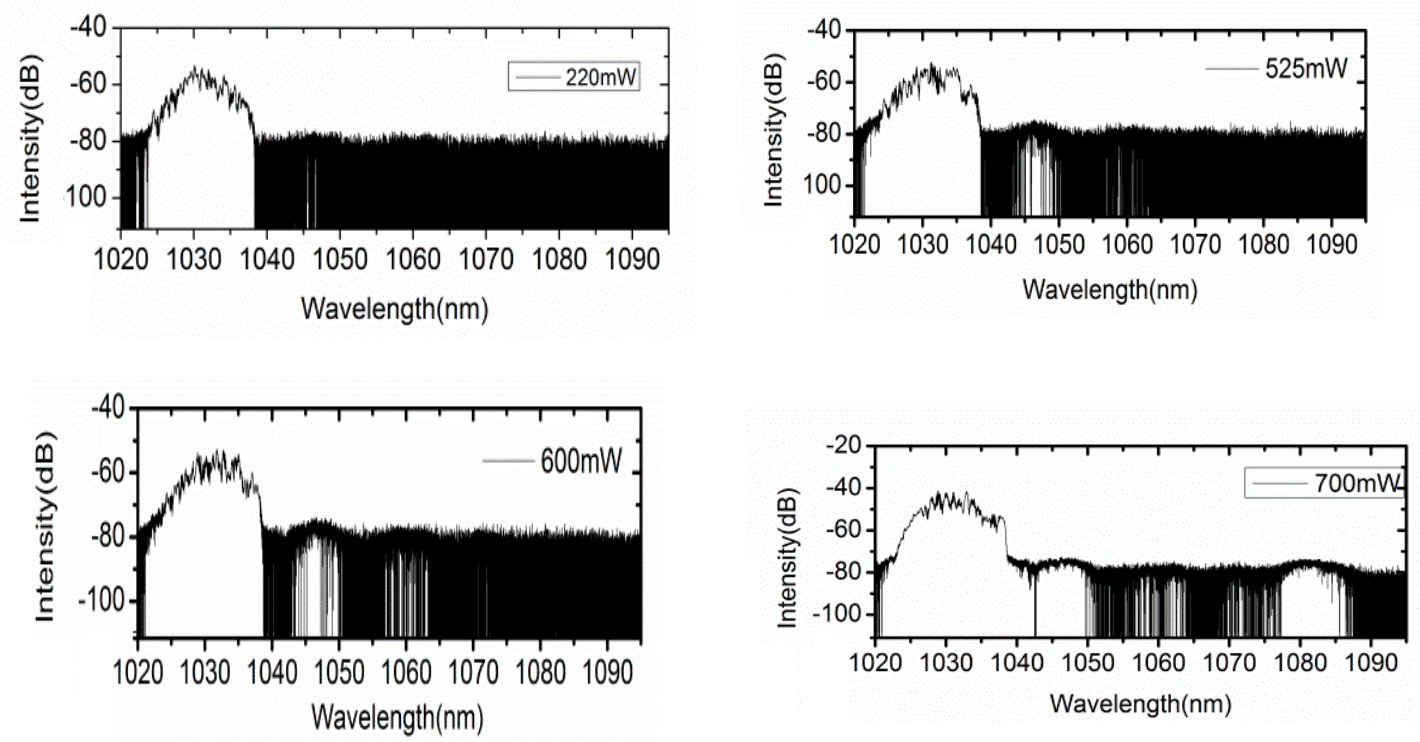

Figure 2. The nonlinear effect of stimulated Raman scattering (SRS) in the fiber preamplifier.

The first stage SCF amplifier's performance is measured with an input power of $600 \mathrm{~mW}$. The output powers of single- and double-pass amplifications are measured, respectively, as shown in Figure 3. Correspondingly, the output power of $4.9 \mathrm{~W}$ and $11.37 \mathrm{~W}$ is obtained with a maximum pump power of 109.2 W. We find that the amplification gain from the single crystal fiber is impressively improved when the laser is operated in double pass. During the double-pass amplification, the maximum amplification gain of 18.95 is obtained.

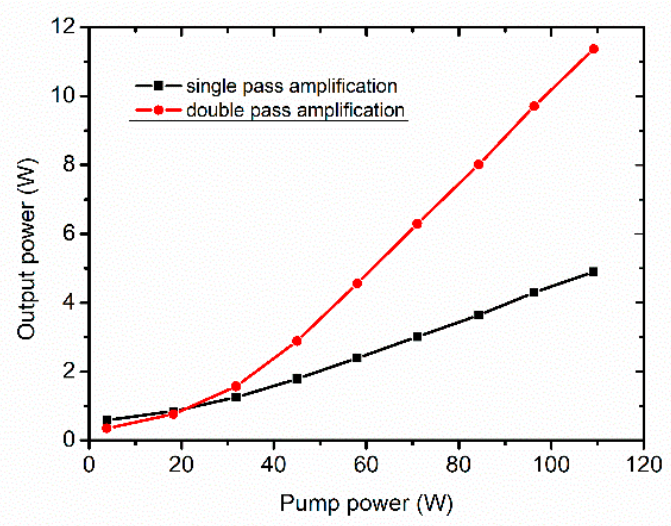

Figure 3. Output power of single- and double-pass amplification of the first stage SCF amplifier. 
After the first stage amplification of SCF amplifier, the laser power is scaled to more than $10 \mathrm{~W}$ at a repetition rate of $250 \mathrm{kHz}$. Then the laser is focused into the second stage amplifier as the signal power with a focused laser diameter of $400 \mu \mathrm{m}$. The amplification outputs of single pass and double pass in the second stage SCF amplifier are measured with different pump powers, as shown in Figure 4. At the maximum pump power, we obtained $34 \mathrm{~W}$ and $41.7 \mathrm{~W}$ in single- and double-pass amplification, respectively. During the double-pass amplification, the thermal lens effect of the SCF has more influence on the gain of the amplification than that of the single-pass amplification. During the double-pass amplification, the saturation process has been observed. To explain this phenomenon, it is speculated that the reabsorption of the amplified laser power will exacerbate the thermal lens effect, which will result in a mode field overlap ratio mismatch between the pump power and the signal power, which influences the amplification gain, and therefore a little saturation is observed. The output beam quality is also measured using Spiricon M2-200s (Ophir, Jerusalem, Israel), as shown in Figure 5. The measured M2 results are 1.328 in the horizontal direction and 1.498 in the vertical direction, respectively. The amplified laser beam density distribution is also detected using a BC106N camera from Thorlabs (Newton, New Jersey, United States), which closes to a Gaussian density distribution shown in the inset of Figure 5.

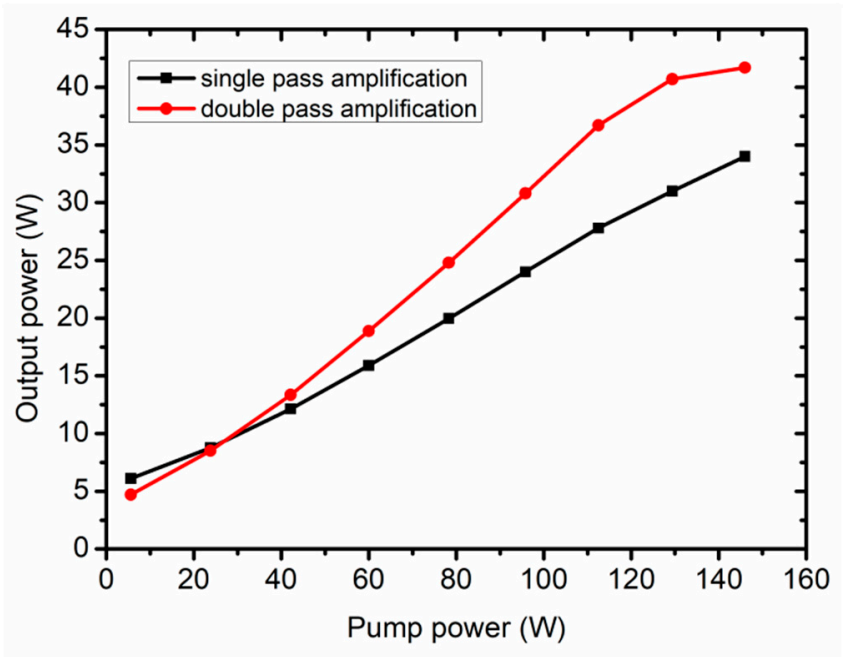

Figure 4. Output power of single- and double-pass amplification of the second stage SCF amplifier.

During the amplification, the spectral gain narrowing effect is measured at different amplification outputs, as shown in Figure 6. The spectra of the fiber preamplifier with a power of $600 \mathrm{~mW}$, the first stage SCF amplifier with a power of $10 \mathrm{~W}$, and the second stage SCF amplifier with a power of $41.7 \mathrm{~W}$ are measured. From the spectra, we find the obvious gain narrowing effect. The gain narrowing issue is caused by the narrow and uneven emission spectrum of the Yb:YAG crystal. Explicitly, the emission spectrum width is less than $8 \mathrm{~nm}$ at the cooling temperature of $20^{\circ} \mathrm{C}$, moreover, the central wavelength component has a higher gain than the off-center wavelengths. As a result, the spectrum width is narrowed when the original seed laser source is amplified. Because the oscillator is a mode-locked fiber laser with positive chirp and spectrum width of $10 \mathrm{~nm}$, the seeder pulse is then stretched to about $40 \mathrm{ps}$ by the $100 \mathrm{~m}$ fiber stretcher. During the amplification, the spectrum is modulated by nonlinear SPM effect and the spectral width is also narrowed by the spectral gain narrowing effect. The spectrum is narrowed to less than $3 \mathrm{~nm}$ when the laser is amplified to $41.7 \mathrm{~W}$. Because the seeder is a chirped pulse output, when the spectrum is narrowed, the pulse width is also narrowed. The autocorrelation curves of the preamplifier, the first- stage SCF amplifier, and the second stage SCF amplifier are measured, as shown in the Figure 7. Correspondingly, the pulse width, with Gaussian fit, is 40.13 ps, $24.86 \mathrm{ps}$, and $16.95 \mathrm{ps}$, respectively. The amplified output pulse spectrum of $3 \mathrm{~nm}$ spectrum width can also sustain the Fourier transform limited pulse duration of $520 \mathrm{fs}$ (Assuming the pulse shape is 
Gaussian shape with a time bandwidth product of 0.441). The SPM is a nonlinear effect which appears during the high energy pulse amplification, especially in the fiber amplifier, where the mode field area is relatively small and interaction length is long. The nonlinear effect will accumulate in the fiber amplifier, which will introduce some nonlinear phase shift during the recompression and lead to the recompressed pulse having some pedestals. As a result of this phenomenon the pulse width cannot be compressed to the Fourier transform limited pulse duration. However, it is possible that the amplified chirped pulse with $\sim 3 \mathrm{~nm}$ spectrum width can also be compressed to femtosecond regime $(<1 \mathrm{ps})$ and even has some pedestals. In some applications, which needs femtosecond laser, this high energy laser source can also be compressed to femtosecond regime by dechirping the laser pulse.

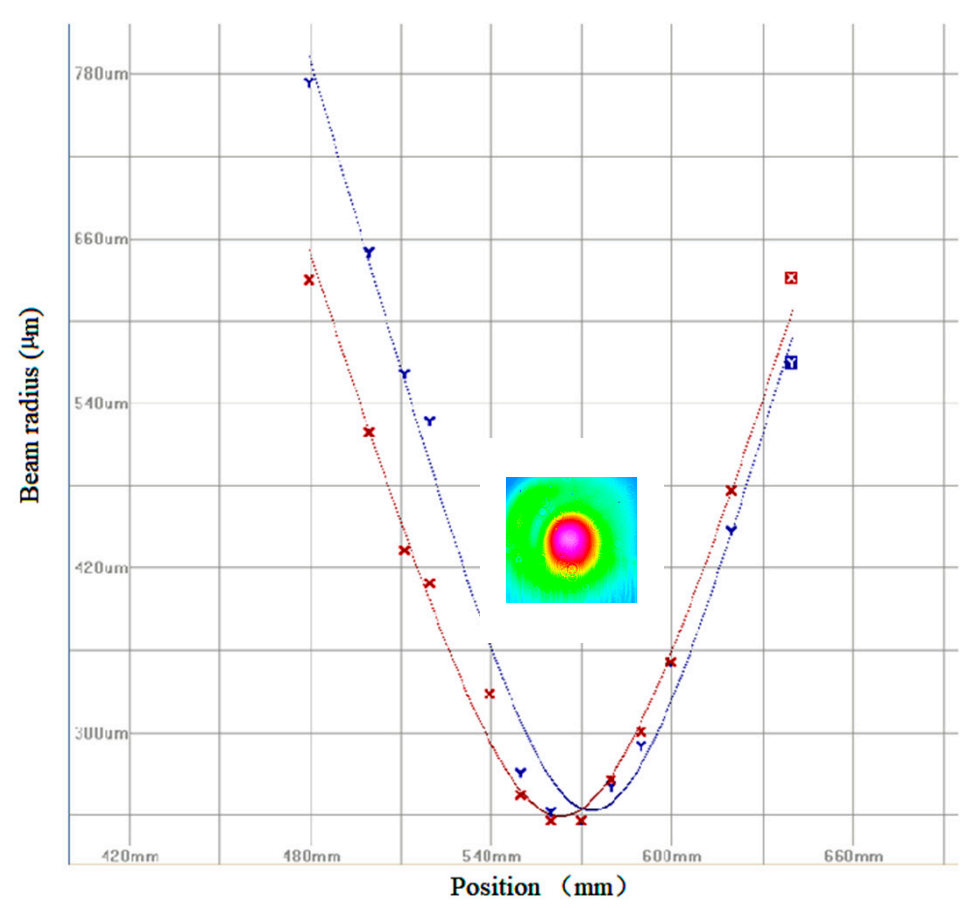

Figure 5. The M2 measurement at the maximum output power.

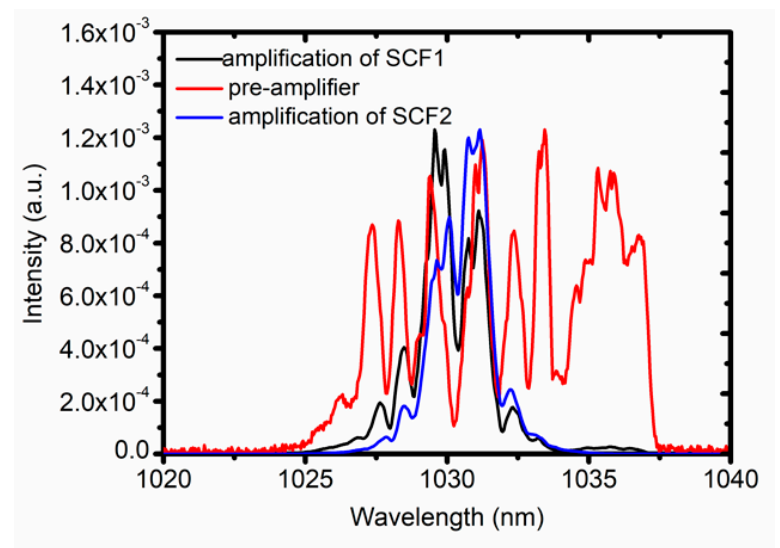

Figure 6. Spectrum measurement of different amplified power outputs.

At the end of this experiment, we also explore the polarization controlled four-pass amplification setup. The setup is shown in Figure 8. Compared to the double-pass configuration, the half-wave plate, polarization beam splitter, and $45^{\circ}$ rotator are inserted into the amplifier to create the polarization controlled four-pass amplification. The amplification performance of single pass, double pass, and four pass is measured, which is shown in Figure 9. The higher gain is obtained during four-pass amplification 
when the signal power of $600 \mathrm{~mW}$ is amplified to more than $20 \mathrm{~W}$. The four-pass amplification scheme has much higher power output during the small signal amplification, a route to get higher gain with a one gain medium. However, it also adds some disadvantages to the system, for example, it is more complicated as there are more lenses for the four-pass amplification, the possibility of an adjustment mistake, the high peak power density will damage the optical components, and furthermore, it is hard to control the amplified beam quality and the four-pass amplification may need more time for system optimization and system integration. Therefore, in the two-stage integration, in order to make the system easy to integrate and ensure the amplified laser has a good beam profile, we do not use the four-pass amplification scheme. For some amplifications, if there is less need for beam quality, the four-pass high gain system can be used in the multistage amplification to obtain much higher output.

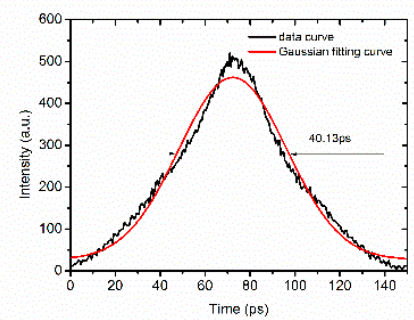

(a)

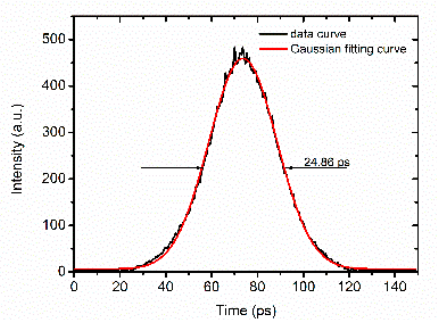

(b)

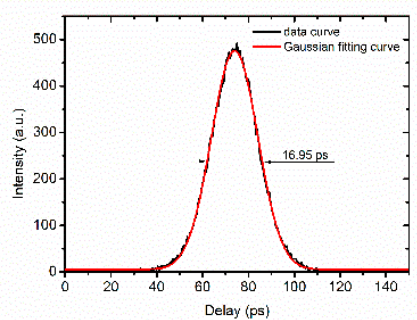

(c)

Figure 7. Pulse width measurement of different amplified power outputs. (a) Autocorrelation curves of the preamplifier; (b) autocorrelation curves of the first- stage SCF amplifier; (c) the second stage SCF amplifier.

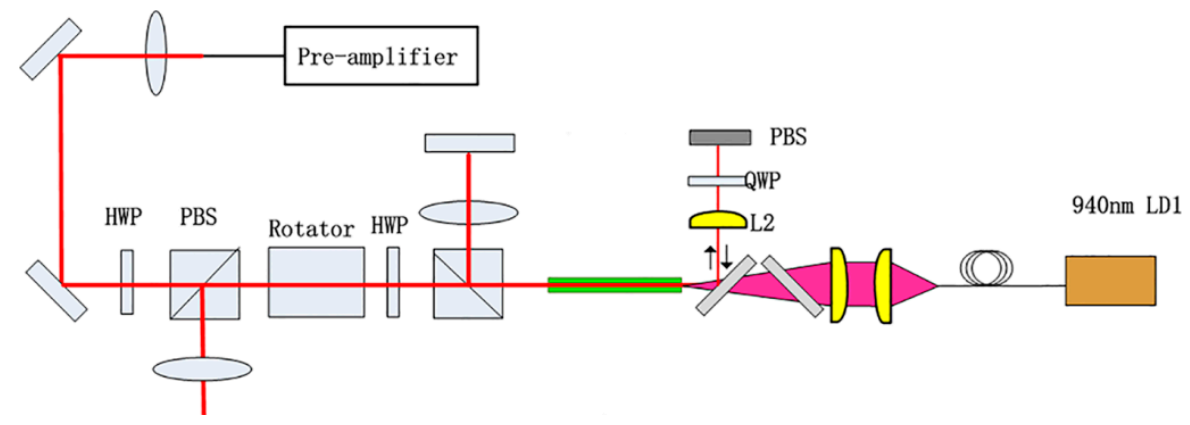

Figure 8. Polarization controlled four-pass amplification.

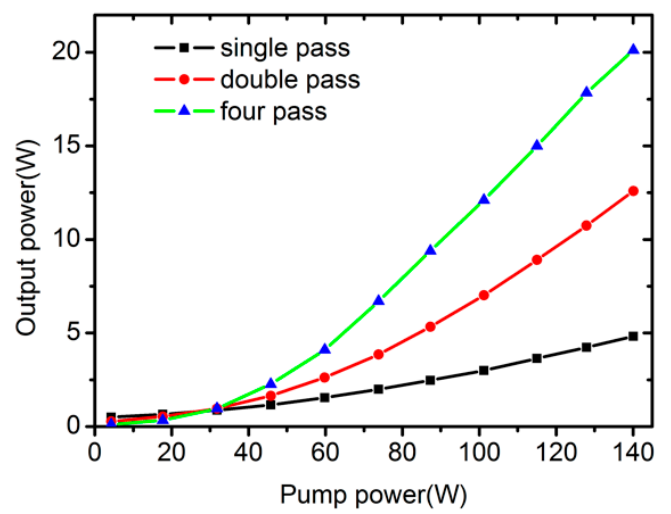

Figure 9. Amplification performance of single-, double-, and four-pass amplification versus the pump power. 


\section{Conclusions}

A high energy ultrashort laser master oscillator pulse amplification (MOPA) system based on a hybrid fiber-single crystal fiber is experimentally studied. During the amplification, the onset of SRS is effectively avoided. By using polarization controlled double-pass amplification of a two-stage Yb:YAG single crystal fiber amplifier, laser pulses with pulse duration of $16.95 \mathrm{ps}$ and high average power of $41.7 \mathrm{~W}$ at the repetition rate of $250 \mathrm{kHz}$ are obtained, corresponding to a peak power of $9.84 \mathrm{MW}$. During the amplification, the spectra gain narrowing effect is also detected. The relation between the spectra width and pulse duration is also experimentally studied. Furthermore, the high-gain four-pass amplification setup of small signal is experimentally studied. The simple and compact laser source will find various applications in micromachining and scientific research.

Author Contributions: For research articles with several authors, a short paragraph specifying their individual contributions must be provided. The following statements should be used "conceptualization, Y.W. (Yishan Wang) and W.Z.; methodology, Z.Y. and F.L.; software, Y.Y.; validation, F.L., Z.L. and Q.L.; formal analysis, F.L.; investigation, F.L.; resources, X.Y., Y.W. (Yufeng Wei); data curation, F.L.; writing—original draft preparation, F.L.; writing-review and editing, Z.Y.; visualization, Y.W. (Yishan Wang); supervision, Z.Y.; project administration, X.Y.; funding acquisition, X.Y.", please turn to the CRediT taxonomy for the term explanation. Authorship must be limited to those who have contributed substantially to the work reported.

Funding: This research was funded by the National Natural Science Foundation of China, (No. 61690222, No. 61805274), the CAS/SAFEA International Partnership Program for Creative Research Teams, and the CAS Light of West China Program (No. XAB2016B21).

Acknowledgments: In this section you can acknowledge any support given which is not covered by the author contribution or funding sections. This may include administrative and technical support, or donations in kind (e.g., materials used for experiments).

Conflicts of Interest: The funders had no role in the design of the study; in the collection, analyses, or interpretation of data; in the writing of the manuscript, or in the decision to publish the results.

\section{References}

1. Kraus, M.; Ahmed, M.A.; Michalowski, A.; Voss, A.; Weber, R.; Graf, T. Microdrilling in steel using ultrashort pulsed laser beams with radial and azimuthal polarization. Opt. Express 2010, 18, 22305-22313. [CrossRef] [PubMed]

2. Gerhard, C.; Druon, F.; Blandin, P.; Hanna, M.; Balembois, F.; Georges, P.; Falcoz, F. Efficient versatilerepetition-rate picosecond source for material processing applications. Appl. Opt. 2008, 47, 967. [CrossRef] [PubMed]

3. Heese, C.; Oehler, A.E.H.; Gallmann, L.; Keller, U. 50-MW, 12-ps Nd:YVO 4, slab amplifier for OPCPA pumping. Conf. Lasers Electro-Opt. 2011. [CrossRef]

4. Boullet, J.; Zaouter, Y.; Desmarchelier, R.; Cazaux, M.; Salin, F.; Saby, J.; Bello-Doua, R.; Cormier, E. High power ytterbium-doped rod-type three-level photonic crystal fiber laser. Opt. Express 2008, 16, 17891-17902. [CrossRef] [PubMed]

5. Malka, D.; Katz, G. An eight-channel C-band demux based on multicore photonic crystal fiber. Nanomaterials 2018, 8, 845. [CrossRef] [PubMed]

6. Chan, H.Y.; Alam, S.U.; Xu, L.; Bateman, J.; Richardson, D.J.; Shepherd, D.P. Compact, high-pulse-energy, high-power, picosecond master oscillator power amplifier. Opt. Express 2014, 22, 21938-21943. [CrossRef] [PubMed]

7. Pierrot, S.; Saby, J.; Bertrand, A.; Liegeois, F.; Duterte, C.; Coquelin, B.; Hernandez, Y.; Salin, F.; Giannone, D. All Fiber High Energy, High Power Picosecond Laser. Conf. Lasers Electro-Opt. 2010. [CrossRef]

8. Liu, T.; Chen, S.; Qi, X.; Hou, J. High-power transverse-mode-switchable all-fiber picosecond MOPA. Opt. Express 2016, 24, 27821-27827. [CrossRef] [PubMed]

9. Teisset, C.; Schultze, M.; Bessing, R.; Häfner, M.; Prinz, S.; Sutter, D.; Metzger, T. 300 W Picosecond Thin-Disk Regenerative Amplifier at $10 \mathrm{kHz}$ Repetition Rate. Adv. Solid State Lasers 2013. [CrossRef]

10. Schmidt, B.E.; Hage, A.; Mans, T.; Légaré, F.; Wörner, H.J. Highly stable, 54 mJ Yb-InnoSlab laser platform at $0.5 \mathrm{~kW}$ average power. Opt. Express 2017, 25, 17549-17555. [CrossRef] [PubMed] 
11. Wang, C.H.; Liu, C.; Shen, L.F.; Zhao, Z.L.; Liu, B.; Jiang, H.B. 1.6 MW peak power, 90 ps all-solid-state laser from an aberration self-compensated double-passing end-pumped Nd:YVO 4 rod amplifier. Appl. Opt. 2016, 55, 2399-2403. [CrossRef] [PubMed]

12. Peng, Y.; Leng, Y.; Chen, J.; Su, H. A 70-W average power sub 100-ps laser based on a fiber and bulk hybrid MOPA configuration. Conf. Lasers Electro-Opt. 2016. [CrossRef]

13. Délen, X.; Zaouter, Y.; Martial, I.; Aubry, N.; Didierjean, J.; Hönninger, C.; Mottay, E.; Balembois, F.; Georges, P. Yb:YAG single crystal fiber power amplifier for femtosecond sources. Opt. Lett. 2013, 38, 109-111. [CrossRef] [PubMed]

14. Saby, J.; Sangla, D.; Deslandes, P.; Salin, F. Non CPA High Energy Picosecond laser based on Single-Crystal Fiber Amplifier. Adv. Solid State Lasers 2014. [CrossRef]

15. Li, F.; Yang, Z.; Lv, Z.G.; Xu, X.H.; Wei, Y.F.; Li, Q.L.; Tang, S.K. Hundred Micro-Joules level High Power Chirped Pulse Amplification of Femtosecond Laser Based on Single Crystal Fiber. Available online: https://ieeexplore.ieee.org/stamp/stamp.jsp?tp=\&arnumber=8166727 (accessed on 21 April 2019).

(C) 2019 by the authors. Licensee MDPI, Basel, Switzerland. This article is an open access article distributed under the terms and conditions of the Creative Commons Attribution (CC BY) license (http://creativecommons.org/licenses/by/4.0/). 